\title{
Network-based Computational Drug Combination Prediction
}

\author{
Fuhai Li, Lei Huang, Jianting Sheng, Stephen Wong
}

\begin{abstract}
Cancers are complex diseases that are regulated by multiple signaling pathways. Patients often acquire resistance to single drug treatment. Use of drug combinations that target multiple parallel pathways is a promising strategy to reduce the drug resistance. Pharmacogenomics big data are being generated to uncover complex signaling mechanisms of cancers and correlate cancer-specific signaling with diverse drug responses. Thus, converting pharmacogenomics big data into knowledge can help the discovery of synergistic drug combination. However, it is challenging and remains an open problem due to the enormous number of combination possibilities and noise of genomics data.
\end{abstract}

\section{INTRODUCTION TO THE TYPE OF PROBLEM IN CANCER}

Cancers are complex diseases regulated by interactions of multiple signaling pathways. Though a number of anti-cancer drugs are currently in use, they not are curative and many are limited by acquired drug resistance. Alternative signaling pathways can maintain tumor development. Use of drug combinations that target multiple parallel pathways [1] is a promising strategy to reduce the drug resistance effect, and improve cancer treatment outcomes. However, it is infeasible to discover drug combinations experimentally due to the enormous number of combination possibilities.

Recent years have seen the explosive generation of large volumes of pharmacogenomics data with the primary goals to uncover the complex signaling mechanisms of cancers and correlate cancer-specific signaling with diverse responses to thousands of drugs. For example, the cancer genome atlas (TCGA) project profiles the genomics of over 10,000 patient samples across over 20 types of cancer [2, 3]. Integrative analyses often indicate multiple subtypes of cancers with complex genomics characteristics $[4,5]$. The Cancer Cell Line Encyclopedia (CCLE) [6], Genomics of Drug Sensitivity in Cancer (GDSC) [7, 8]. The Connectivity Map (CMAP) [9] generated gene expression signature data of different cell lines under various perturbations by 1,039 small molecules. These data have since been scaled over 1,000 times (including 5585 drugs and bioactives, and 20,000+ genetic reagents (targets)) and are publicly available at (http://www.lincscloud.org/) (LINCS: Library of Integrated Network-based Cellular Signatures).

Though rich information is embedded in the pharmacogenomics big data, it remains an open problem to convert that big data to knowledge for discovery drug

F. Research supported by NIH U54 CA149196 and CPRIT RP110532.

F. L., L. H., S. Wong. are with the NCI Center for Modeling Cancer Development, Department of Systems Medicine and Bioengineering, Houston Methodist Hospital Research Institute; Weill Cornell Medical College of Cornell University, Houston, TX, 77030, U.S.A. (e-mails: fli@tmhs.org; lhuang@tmhs.org; jsheng@tmhs.org; stwong@tmhs.org; corresponding author to S. W.) combination. Some of the challenges are as follows. First, there are millions of possible pair-wise drug combinations of even 1000 drugs. Secondly, the genomics profile data are noisy for both cancer patients, and compounds and genetic reagents from CMAP; and 2) the selection of synergistic drug combinations from millions of possible combinations is difficult. Therefore, novel data-driven approaches are needed urgently to integrate genomics profiles of cancers and thousands of drugs and molecules, and genetic reagents to predict the synergistic drug combinations.

Network-based drug discovery is touted as the next drug discovery paradigm [10]. Diseases are often regulated by complex signaling networks, and multi-drugs and multitargets are often associated to form a big drug-drug, and drug-target network. On the other hand, there are rich computational resources to support the network analysis and visualization. In this study, we introduce a network-based computational approach for synergistic drug combination prediction [11].

\section{Illustrative RESUltS OF APPLICATION OF METHODS}

The proposed drug combination prediction approach was evaluated on lung adenocarcinoma. The genomics profiles of lung adenocarcinoma patients were collected from GEO (Gene Expression Omnibus) (GDS3257) to reconstruct the lung adenocarcinoma-specific signaling network. The genomics profiles of 1309 drugs and compounds were obtained from CMAP to build the drug network modules. Three drugs, Gefitinib, Paclitaxel and LY-294002, were used as the anchor drugs, and the optimal drugs combining with them are predicted (ranked). Through literature review as the validation, among the top 50 predicted drug pairs, 9, 8, and 3 drug pairs have been reported to be effective respectively [11]. This success rate is significantly higher compared with other drug combination methods and random selection [11]. Figure 1 shows the distribution of Gefitinib and Paclitaxel targets on the reconstructed lung adenocarcinoma disease

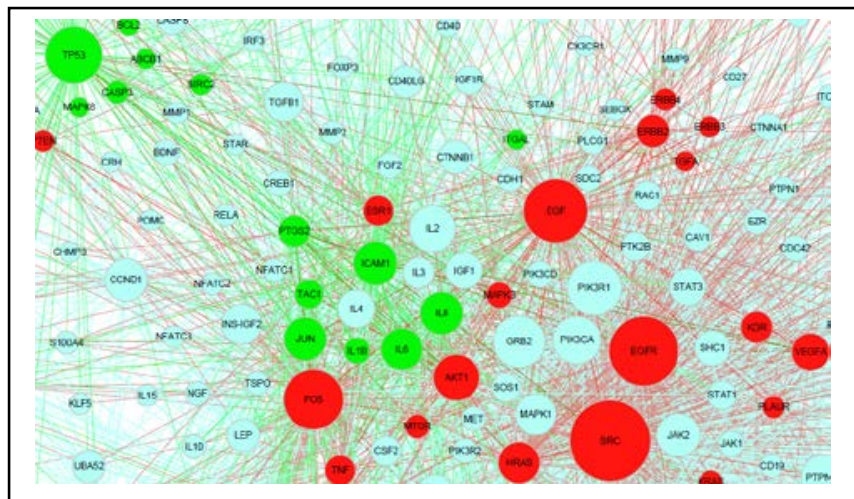

Figure 1. Target distribution of (red) Gefitinib and (green) Paclitaxel on the reconstructed lung adenocarcinoma dsease signaling network. 
bioRxiv preprint doi: https://doi.org/10.1101/049015; this version posted April 16, 2016. The copyright holder for this preprint (which was not certified by peer review) is the author/funder, who has granted bioRxiv a license to display the preprint in perpetuity. It is made available under aCC-BY-ND 4.0 International license.

signaling network, which indicates the potential mechanism of action of the drug combination.

\section{QUicK GUIDE TO THE MethodS}

\section{A. Major assumptions of the model}

The proposed approach was designed based on the following major assumptions. First, disease signaling networks have alternative signaling modules. Secondly, drugs can inhibit multiple targets on the disease signaling network. Thirdly, effective drug combinations can inhibit alternative signaling modules simultaneously.

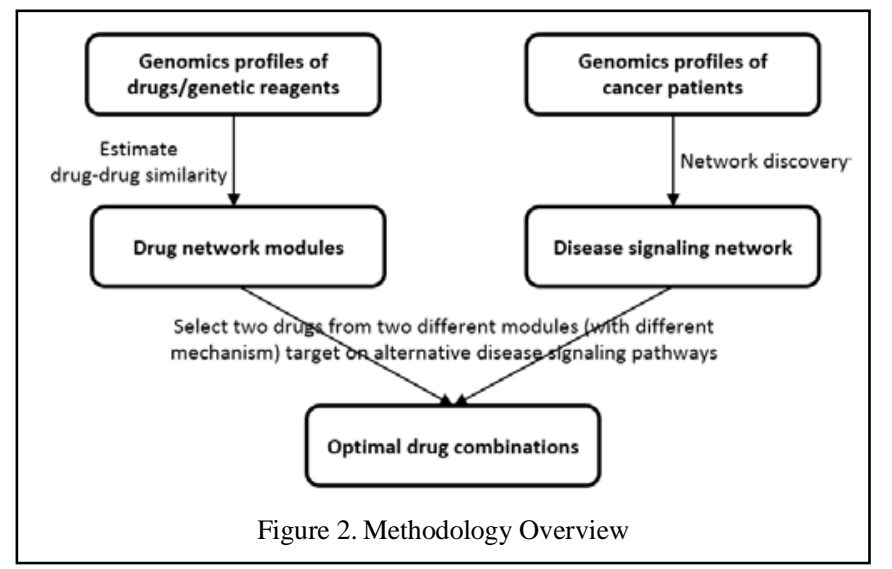

\section{B. Methodology Overview}

Figure 2 shows the methodology overview. In general, the drug-drug similarity (distance) is estimated based on the genomics profiles of drugs from the CMAP database [12]. Other information can also be integrated to refine the drugdrug similarity, e.g., the drug-target interaction information in STITCH [13]. Then drugs are connected into a drug-drug network given a similarity threshold, which is partitioned into drug network modules using the network analysis [12] or clustering approaches [11]. Different drug network modules might share the common mechanism of action, and their targets can indicate the inhibited signaling modules of diseases. The drug target information can be obtained from the following database, e.g., DrugBank [14], STITCH [13], NPC browser [15]. The disease signaling network can be reconstructed [16, 17] by integrating disease genomics data and protein interactome data, e.g., BioGRID [18], Biocarta [19], Reactome [20], HPRD [21], and KEGG [22]. Drugs (from different drug network modules) are combined to disrupt disease signaling network modules using the network centrality metrics [23, 24].

\section{Network analysis and visualization tools}

There are rich computational resources of network-based approaches that can be used for biomarker and drug discovery. Some widely used network analysis tools are, e.g., igraph in R (http://igraph.org/r/), NetworkX in Python (https://networkx.github.io/) for network structure analysis, and, e.g., Cytoscape (http://www.cytoscape.org/), and D3.js (http://d3js.org/) for network visualization.

\section{ACKNOWLEDGMENT}

We would like to thank the bioinformatics group of the department of Systems Medicine and Bioengineering for their helpful discussions.

\section{REFERENCES}

[1] Jia, J., et al., Mechanisms of drug combinations: interaction and network perspectives. Nat Rev Drug Discov, 2009. 8(2): p. 111-28.

[2] Weinstein, J.N., et al., The Cancer Genome Atlas Pan-Cancer analysis project. Nat Genet, 2013. 45(10): p. 1113-20.

[3] TCGA-web, https://tcga-data.nci.nih.gov/tcga/tcgaHome2.jsp.

[4] Hofree, M., et al., Network-based stratification of tumor mutations. Nat Methods, 2014. 10(11): p. 1108-15.

[5] Kandoth, C., et al., Integrated genomic characterization of endometrial carcinoma. Nature, 2013. 497(7447): p. 67-73.

[6] Barretina, J., et al., The Cancer Cell Line Encyclopedia enables predictive modelling of anticancer drug sensitivity. Nature, 2012. 483(7391): p. 603-7.

[7] Garnett, M.J., et al., Systematic identification of genomic markers of drug sensitivity in cancer cells. Nature, 2012. 483(7391): p. 570-5.

[8] Yang, W., et al., Genomics of Drug Sensitivity in Cancer (GDSC): a resource for therapeutic biomarker discovery in cancer cells. Nucleic Acids Res, 2013. 41(Database issue): p. D955-61.

[9] Lamb, J., et al., The Connectivity Map: using gene-expression signatures to connect small molecules, genes, and disease. Science, 2006. 313(5795): p. 1929-35.

[10] Barabasi, A.L., N. Gulbahce, and J. Loscalzo, Network medicine: a network-based approach to human disease. Nat Rev Genet, 2011. 12(1): p. 56-68.

[11] Huang, L., et al., DrugComboRanker: drug combination discovery based on target network analysis. Bioinformatics, 2014. 30(12): p. i228-i236.

[12] Iorio, F., et al., Discovery of drug mode of action and drug repositioning from transcriptional responses. Proc Natl Acad Sci U S A, 2010. 107(33): p. 14621-6.

[13] Kuhn, M., et al., STITCH 3: zooming in on protein-chemical interactions. Nucleic Acids Res, 2012. 40(Database issue): p. D87680.

[14] Wishart, D.S., et al., DrugBank: a knowledgebase for drugs, drug actions and drug targets. Nucleic Acids Res, 2008. 36(Database issue): p. D901-6.

[15] Huang, R., et al., The NCGC pharmaceutical collection: a comprehensive resource of clinically approved drugs enabling repurposing and chemical genomics. Sci Transl Med, 2011. 3(80): p. 80ps16.

[16] 16. Barrenas, F., et al., Highly interconnected genes in diseasespecific networks are enriched for disease-associated polymorphisms. Genome Biol, 2012. 13(6): p. R46.

[17] Chuang, H.Y., et al., Network-based classification of breast cancer metastasis. Mol Syst Biol, 2007. 3: p. 140.

[18] Stark, C., et al., BioGRID: a general repository for interaction datasets. Nucleic Acids Res, 2006. 34(Database issue): p. D535-9.

[19] BioCarta, http://www.biocarta.com/.

[20] Matthews, L., et al., Reactome knowledgebase of human biological pathways and processes. Nucleic Acids Res, 2009. 37(Database issue): p. D619-22.

[21] Keshava Prasad, T.S., et al., Human Protein Reference Database-2009 update. Nucleic Acids Res, 2009. 37(Database issue): p. D76772.

[22] Kanehisa, M., et al., KEGG for linking genomes to life and the environment. Nucleic Acids Res, 2008. 36(Database issue): p. D4804.

[23] Brandes, U. and D. Fleischer, Centrality measures based on current flow, in Proceedings of the 22nd annual conference on Theoretical Aspects of Computer Science. 2005, Springer-Verlag: Stuttgart, Germany.

[24] Page, L., et al., The PageRank Citation Ranking: Bringing Order to the Web. 1999, Stanford InfoLab. 